\title{
RECENT PROGRESS ON RELIABILITY ASSESSMENT OF LARGE-EDDY SIMULATION
}

\author{
BERNARD J. GEURTS ${ }^{(1,2)}$ AMIRREZA ROUHI ${ }^{(3,4)}$ UGO PIOMELLI $^{(3)}$
}

(1) Multiscale Modeling and Simulation, Department of Applied Mathematics, University of Twente, P.O. Box 217, 7500 AE Enschede, The Netherlands

(2) Multiscale Physics of Energy Systems, Center for Computational Energy Research , Faculty of Applied Physics, Eindhoven University of Technology, P.O. Box 513, 5600 MB Eindhoven, The Netherlands

(3) Department of Mechanical and Materials Engineering, Queen's University, Kingston

(Ontario) K7L 4L9, Canada

(4) Department of Mechanical Engineering, University of Melbourne, Victoria 3010, Australia

b.j.geurts@utwente.nl

\begin{abstract}
Reliability assessment of large-eddy simulation (LES) of turbulent flows requires consideration of errors due to shortcomings in the modeling of sub-filter scale dynamics and due to discretization of the governing filtered Navier-Stokes equations. The Integral Length-Scale Approximation (ILSA) model is a pioneering sub-filter parameterization that incorporates both these contributions to the total simulation error, and provides user control over the desired accuracy of a simulation. It combines an imposed target for the 'sub-filter activity' and a flow-specific length-scale definition to achieve LES predictions with pre-defined fidelity level. The performance of the 'global' and the 'local' formulations of ILSA, implemented as eddy-viscosity models, for turbulent channel flow and for separated turbulent flow over a backward-facing step are investigated here. We show excellent agreement with reference direct numerical simulations, with experimental data and with predictions based on other, well-established sub-filter models. The computational overhead is found to be close to that of a basic Smagorinsky sub-filter model.
\end{abstract}

\section{Introduction}

Large-eddy simulation (LES) of turbulent flow has a long and rich history in which already during the 1960s first parameterizations, such as Smagorinsky's eddy-viscosity model (Smagorinsky, 1963) were proposed to capture the effects of localized turbulent motions on the large energycarrying scales. The coarsening length-scale of choice was directly linked to the mesh-size in the computational grid, often chosen as the cube-root of the volume of a grid cell (Schumann, 1975). However, the computational grid is often defined prior to any flow simulation and a direct, quantitative link between the grid-based local coarsening length-scale and the actual local flow is not made. Moreover, while coarsening is helpful in reducing the computational effort required for a simulation of a particular flow, it also introduces uncertainty regarding the accuracy of the achieved results (Pope, 2000; Geurts, 2003). Based on these observations two aspects take a central role in LES research, i.e., (i) developing a flow-related length-scale distribution allowing efficient as well as grid-independent LES, and (ii) achieving a clear estimation/control of the level 
of uncertainty in the coarsened predictions. These are crucial timely pacing items in LES research that are in the focus of this paper. We review the recent ILSA proposal (Integral Length-Scale Approximation) which is a first framework that can address both aspects systematically, closely following Piomelli et al (2015) and Rouhi et al. (2016).

Computational assessment and comparison of large-eddy simulation methods allows to address reliability issues in LES. The so-called error-landscape approach (Meyers et al., 2003) gives a direct measurement of the difference between a particular LES and the corresponding DNS (Meyers et al, 2007) or experimental (Meyers et al., 2010) findings. This approach makes it possible to identify partial error cancellation arising from the interaction between modeling and discretization error effects (Vreman, et al., 1994). Moreover, it suggests an 'optimal refinement strategy' yielding minimal error at given computational cost. This is a first ingredient for the ILSA method. We show results of the error-landscape method for homogeneous isotropic decaying turbulence using a Smagorinsky sub-filter model. By systematically varying the simulation resolution and the Smagorinsky coefficient, one can determine parameter regions for which a desired number of flow properties is simultaneously predicted with approximately minimal error (Meyers et al., 2006). The dynamic effects of discretization errors are particularly important at marginal spatial resolution as may be found in parts of flow domains with complex flow behavior. Under marginal resolution conditions the asymptotic error behavior as expressed by the order of the spatial discretization is no longer characteristic for the total dynamic consequences of discretization errors (Geurts, 2006).

The computational grid for LES is often defined independent of the flow. Correspondingly, also the grid-based local coarsening length-scale is decoupled from the actual local flow. This clearly is not optimal for accuracy and efficiency. In fact, LES coarsening could in principle differ from location to location and from time to time, in response to local turbulence levels and variations in length- and time scales while the flow develops. Such technique would allow for larger grid spacing in regions of rather quiescent flow and adopt higher resolution where required by the locally more detailed flow (Boersma et al., 1997). Recently, in Piomelli \& Geurts (2010) and Piomelli et al. (2015) an alternative coarsening length-scale was put forward for LES, based on flow physics rather than on the grid scale. This idea was implemented in the form of an eddyviscosity model for which the turbulent eddy-viscosity was based on an estimate of the local integral length-scale.

The model coefficient in the ILSA proposal is specified either through minimizing the error in a fluid mechanical property (e.g., skin friction coefficient or turbulent kinetic energy), or through a user-defined LES resolution measure, following the concept of sub-filter activity as suggested by Geurts \& Fröhlich (2002). Model parameter optimization can be inferred computationally from exploratory coarser simulations, following the SIPI (Successive Inverse Polynomial Interpolation) error minimization (Geurts \& Meyers, 2006). Combined, ILSA is a first, complete formulation in which the issue of LES reliability for a particular flow is put at the central place in the computational framework that it deserves.

In this paper we review the ILSA modeling strategy and discuss the development and testing of the new model for turbulent channel flow at high Reynolds numbers. Moreover, new results for key quantities of turbulent flow over a backward-facing step are presented, showing that the new eddy-viscosity model compares closely with experimental data by Vogel \& Eaton (1985). ILSA does not require the introduction of any ad hoc user-defined parameters, other than the target subfilter activity, i.e., the level of LES resolution that is 'deemed acceptable' by the user prior to an actual simulation. We investigate the spatially non-uniform flow coarsening achieved using ILSA in boundary layers and for the backward-facing step and show that it yields smooth turbulent eddy-viscosity distributions, despite the sharply refined grid, avoiding any numerical jumps that 
appear in traditional grid-based formulations. In its original formulation, ILSA is an example of a 'multi-resolution' method in which effectively findings at coarse grids are integrated to achieve a solution-specific length scale for subsequent high-fidelity production runs. This method allows to separate the problem of controlling discretization error effects in a coarsened flow model from that of sub-filter modeling errors in the final coarse solution. The formulation supports the notion of grid-independent LES, in which a prespecified reliability measure is used to determine the local coarsening length-scale. This is basic to achieving a priori error control.

The organization of this paper is as follows: In Section 2 we briefly review reliability issues in LES and identify the main limiting factors that determine the overall reliability of LES predictions. Basic ILSA is presented in Section 3 in which first the original 'global' ILSA is formulated. This highlights the use of 'pre-cursor' simulations on coarse grids to achieve a computational optimization of the effective model parameter, ahead of any high-fidelity production runs, exploiting the SIPI algorithm. In Section 3 we discuss the 'local' ILSA extension, which rests on the same concept but avoids pre-cursor simulations by quantifying the sub-filter activity directly in terms of an invariant of the sub-filter tensor. Turbulent channel flow at high Reynolds numbers was a first successful application of ILSA - reviewed in Section 4. Section 5 presents results for turbulent backward-facing step flow, closely following Rouhi et al. (2016), showing high-accuracy predictions of the separated flow region, fully consistent with experimental findings. Summarizing remarks are collected in Section 6.

\section{Reliability issues in large-eddy simulation}

In this Section we briefly review the main components that make up the total simulation error in LES and discuss the error-landscape approach to visualize interacting error contributions, which are basic to the fact that the total simulation error is not simply the sum of the absolute values of its components. In fact, partial error cancellation may occur, giving rise to particular LES paradoxes (Geurts, 1999; Geurts 2002).

A standard formulation for LES assumes a spatial convolution filter with an effective width $\Delta$, coupling the unfiltered Navier-Stokes solution to the filtered solution. In this paper we work with incompressible flows, governed by conservation of mass and momentum respectively,

$$
\begin{aligned}
& \partial_{j} \bar{u}_{j}=0 \\
& \partial_{t} \bar{u}_{i}+\partial_{j}\left(\bar{u}_{i} \bar{u}_{j}\right)+\partial_{i} \bar{p}-\frac{1}{R e} \partial_{j j} \bar{u}_{i}=-\partial_{j}\left(\bar{u}_{i} u_{j}-\bar{u}_{i} \bar{u}_{j}\right)
\end{aligned}
$$

where the overbar denotes the filtered variable. Here, we use Einstein's summation convention and use $p$ for the pressure and $\boldsymbol{u}$ for the velocity field. Time is denoted by $t$ and partial differentiation with respect to the $j$-th coordinate by the subscript $j$. Relevant length- $(L)$ and velocity $(U)$ scales, and the constant density and kinematic viscosity $(v)$ are used to make the equations dimensionless and define the Reynolds number $R e=U L / v$. On the left-hand side we observe the incompressible Navier-Stokes formulation in terms of the filtered variables. On the right hand side the filtered momentum equation has a non-zero contribution expressed in terms of the divergence of the sub-filter stress tensor

$$
\tau_{i j}=\overline{u_{i} u_{j}}-\bar{u}_{i} \bar{u}_{j}
$$

The sub-filter tensor expresses the central 'closure problem' in LES, as it requires both the filtered as well as the unfiltered representation of the solution. Since only the filtered solution is available

$$
N S(\mathbf{u})=0 \quad \Rightarrow \quad N S(\overline{\mathbf{u}})=-\nabla \cdot \tau(\mathbf{u}, \overline{\mathbf{u}}) \Leftarrow-\nabla \cdot M(\overline{\mathbf{u}})
$$


in LES, the next step in modeling the coarsened turbulent flow is to propose a sub-filter model $M$ in terms of the filtered solution only. In short-hand notation this may be expressed as

Here, the unfiltered problem consists of finding $\mathbf{u}$ from the problem $N S(\mathbf{u})=0$, while after filtering the unclosed problem $N S(\overline{\mathbf{u}})=-\nabla \cdot \tau(\mathbf{u}, \overline{\mathbf{u}})$ is replaced by $N S(\mathbf{v})=-\nabla \cdot M(\mathbf{v})$, which is the closed problem corresponding to the sub-filter model $M$ from which the approximate filtered solution $\mathbf{v}$ can be obtained. Numerous sub-filter models have been proposed for LES. In this paper we restrict ourselves to eddy-viscosity models, in which the anisotropic part of sub-filter stress tensor is given by $\tau_{i j}^{a}=-2 \nu_{s f s} S_{i j}$, where $S_{i j}$ denotes the rate of strain tensor of the filtered velocity field, i.e., the symmetric part of the velocity gradient, and $v_{s f s}$ is the sub-filter scale eddy viscosity.

A central premise of numerical simulation asserts that the solution to a given PDE problem should be obtained accurately and efficiently, while simultaneously, a close upper-bound for the error should be estimated. In the context of LES this not only implies a study of the effects of numerical discretization errors on the dynamics of the simulated solution, but also includes the role of the model for the sub-filter stress tensor as well as the interaction between these two basic sources of error (Geurts, 1999; Nicoud et al., 2001; Van der Bos et al., 2007). In principle, the role of the numerical discretization in an academic LES setting can be fully controlled. In fact, the spatial filtering creates a smoothing of the problem and also separates scales larger than the filter-width from scales smaller than this filter-width. The computational grid provides an additional (local) length scale and theoretically it is sufficient to require that the sub-filter resolution, i.e., the ratio between the filter-width and the mesh-size is sufficiently large. In that asymptotic, theoretical, regime good spatial discretization methods should converge rapidly with grid refinement, while keeping the filter-width constant. This would correspond to a grid-independent LES solution, characteristic of the adopted sub-filter model. However, in practice the computational costs of simulating a flow on $N^{3}$ grid points, using an explicit time-stepping method, scales $\sim N^{4}$ with $N$ the number of grid points along a coordinate direction. This cost hinders the sub-filter resolution to be very large in practice, suggesting that there is likely a large role of the numerical method in capturing the actual LES solution (Geurts \& Van der Bos, 2005). Hence, at practically feasible, marginal resolution, both the selected sub-filter model as well as the adopted spatial discretization method can have a significant influence on the simulated dynamics. Together, these influences give rise to the total simulation error. 
Since the modeling and discretization error effects can partially counteract each other it is not straightforward to assess the overall simulation error in a given flow property. Instead, one can resort to a computational assessment of the simulation error for selected cases. This is known as the error-landscape approach. In Figure 1 we show such an error-landscape for LES of homogeneous isotropic turbulence, based on the Smagorinsky model. The error is based on the relative deviation of the turbulent kinetic energy between, on the one hand, a particular LES (at given spatial resolution $N$ and value of the Smagorinsky coefficient $C_{S}$ ) and, on the other hand, the underlying direct numerical simulation. Each dot on the error-landscape surface denotes the error in a particular LES. At zero Smagorinsky coefficient, e.g., the LES corresponds to a 'nomodel' or under-resolved simulation. We observe that the error decreases rapidly and smoothly with increasing spatial resolution, indicating convergence toward DNS predictions at high enough spatial resolution. Moreover, we notice that at fixed, coarse, spatial resolution $N$ and sufficiently large values of the Smagorinsky coefficients, also rather large errors arise. In between the "no model' case and a very large $C_{S}$ there appears a minimum in which possible partial cancellation of modeling and discretization error effects is achieved optimally at that value of grid resolution $N$. This would yield the lowest total simulation error at the corresponding computational cost. The optimal refinement strategy can be inferred by determining these minima as function of $N$. Knowledge about such error behavior can be used to classify errors due to numerical dissipation and sub-filter contributions (Van der Bos \& Geurts, 2010). Strictly speaking, the optimal refinement strategy can be inferred only after a database of LESs is collected - the optimal 
Smagorinsky coefficient at given spatial resolution is a quantity that currently cannot be predicted in advance theoretically (Klein et al., 2008).

A computational estimate of the optimal Smagorinsky coefficient at given spatial resolution can be obtained at modest additional cost using the SIPI method (Successive Inverse Polynomial Interpolation) (Geurts \& Meyers, 2006). At given $N$ this method takes error levels at three prior simulations using different $C_{S}$ values, and, via quadratic interpolation, progresses to converge $C_{S}$ to achieve the error minimum. SIPI requires about five LESs to approximate the optimal Smagorinsky coefficient with good accuracy. Since the dependence of the optimal Smagorinsky coefficient on the spatial resolution is quite modest, one may proceed in two steps. First, at coarse resolution the optimal Smagorinsky coefficient is determined. Subsequently, at finer resolution, production simulations can be executed with this optimal coarse grid value. This approach is also basic to the original ILSA model to which we turn next.

\section{ILSA - Integral Length-Scale Approximation}

We review the length-scale definition for LES based on the resolved turbulent kinetic energy (TKE) and its dissipation. Rather than working with a grid-based length-scale, as in traditional LES, referring to sub-grid scales, we propose a flow-specific length-scale distribution defining the filter-width and hence refer to the LES approach as modelling the sub-filter scales. An important benefit of this distinction is the fact that by resolving the new length-scale on the computational grid, a grid-independent LES is feasible, allowing to discriminate between discretization and sub-filter modeling contributions to the overall error.

The global ILSA model is an eddy-viscosity model in which the anisotropic part of the sub-filter stress tensor is given by $\tau_{i j}^{a}=-2 \nu_{s f s} S_{i j}$ with turbulent eddy-viscosity defined as

$$
\nu_{s f s}=\left(C_{m} \Delta\right)^{2}|\bar{S}| \equiv\left(C_{m} C_{\Delta} L\right)^{2}|\bar{S}| \equiv\left(C_{k} L\right)^{2}|\bar{S}|
$$

where $C_{k}=C_{m} C_{\Delta}$ is referred to as the 'effective model coefficient', and the filter-width $\Delta$ is expressed as a fraction of the local integral length-scale, $\Delta=C_{\Delta} L$, inferred from

$$
L=\frac{\left\langle K_{r e s}\right\rangle}{\left\langle\epsilon_{t o t}\right\rangle}
$$

where the resolved turbulent kinetic energy (TKE) and total dissipation rate are given by

$$
K_{\text {res }}=\frac{1}{2} \bar{u}_{i}^{\prime} \bar{u}_{i}^{\prime} \quad ; \quad \varepsilon_{\text {tot }}=2\left(\nu+\nu_{s f s}\right) \bar{S}_{i j}^{\prime} \bar{S}_{i j}^{\prime}
$$

in terms of resolved velocity fluctuations and the corresponding rate-of-strain tensor. Using the resolved TKE rather than the total one does not affect the estimated length-scale significantly (Piomelli et al. 2015). The choice to use the integral length scale $L$ implies that the local LES resolution adapts itself dynamically to the turbulence characteristics of the flow. The local grid resolution $h$ should at least resolve the integral length scale $L$, i.e., $L / h>>1$. By selecting $h$ appropriately, an approximately grid-independent LES prediction may be obtained. Moreover, variations in $L$ automatically can be used to generate (adaptive) non-uniform grids on which to simulate the turbulent flow at hand (Boersma et al., 1997).

Aside from the local integral length-scale $L$, a key ingredient of the ILSA model is that adaptations in the effective model coefficient are made consistent with a measure toward explicit LES resolution control. This way, the effective model coefficient $C_{k}$ should be obtained in response 
to the flow characteristics. For this purpose the concept of sub-filter activity (Geurts \& Fröhlich, 2002 ) is used. We build the dimensionless sub-filter activity in terms of the turbulent and molecular dissipation rates as

$$
s_{\varepsilon}=\frac{\left\langle\varepsilon_{s f s}\right\rangle}{\left\langle\varepsilon_{s f s}+\varepsilon_{\nu}\right\rangle} \quad ; \quad\left\langle\varepsilon_{s f s}\right\rangle=\left\langle 2 \nu_{s f s} \bar{S}_{i j}^{\prime} \bar{S}_{i j}^{\prime}\right\rangle
$$

In the original formulation of ILSA called 'global ILSA' (Piomelli et al. 2015), the averaging used to calculate the sub-filter activity was carried out over the entire computational domain and time, implying that the global contribution of sub-filter scales was assigned to $C_{k}$ as a constant model coefficient. If the sub-filter activity is close to 0 then the relevance of the flow scales that are still unresolved compared to the local integral scale is rather modest - we are close to resolution conditions needed for a direct numerical simulation, implying that remaining errors will be mainly of discretization nature. On the contrary, if the sub-filter activity is approaching 1, almost all dynamic scales are unresolved compared to the local integral scale and the sub-filter modeling error will be important as well. By controlling the allowed value of the sub-filter activity, we may dynamically adapt the effective model coefficient and hence exert some control over the dominant source and magnitude of the total simulation error. In fact, if we put $s_{\varepsilon}=\delta$ for some value $\delta$ then we infer that locally

$$
\nu_{s f s}=C_{k}^{2} L^{2}|S| \leq \frac{\delta \nu}{1-\delta}
$$

In deriving this, we allowed the turbulent viscosity to be taken out of the averaging operator. This approach yields the effective model coefficient in response to the integral length-scale, the size of the rate of strain tensor and the molecular viscosity. Taking $\delta$ small enough, i.e., $C_{k}^{2} L^{2}$ small, the LES can, in principle, be made as accurate as desired by pushing resolution conditions toward DNS. This approach is conceptually related to the famous 'Pope 80\% rule' (Pope, 2000) in which it is postulated that accurate LES requires the local filter-width to be such that the resolved turbulent kinetic energy is at least $80 \%$ of the total turbulent kinetic energy. Likewise, requiring $s_{\varepsilon}=\delta$ we inherit a dynamic model response compliant with this accuracy condition. Since the subfilter activity is not known a priori as function of $C_{k}$, the assignment of the correct level of subfilter activity $\delta$ is actually an inverse problem. This can be solved, approximately, computationally by simulating a particular flow on a coarse mesh at a range of values for the effective model coefficient and determining the sub-filter activity for each value of $C_{k}$. Once such pre-cursor simulations have been done, one can set a suitable approximate value for the effective model coefficient such that the sub-filter activity level remains near the selected 'target' value.

A modification of the ILSA model can be devised in which the local contribution of sub-filter scales is employed and the spatially and temporally non-uniform $C_{k}$ can be found without the precursor simulations. This is known as "local ILSA", to which we turn next. Instead of measuring sub-filter activity in terms of the turbulent dissipation rates, we consider invariants of the subfilter stresses directly. Following Rouhi et al., (2016) we introduce

$$
s_{\tau}=\left(\frac{\left\langle\tau_{i j}^{a} \tau_{i j}^{a}\right\rangle}{\left\langle\left(\tau_{i j}^{a}+R_{i j}^{a}\right)\left(\tau_{i j}^{a}+R_{i j}^{a}\right)\right\rangle}\right)^{1 / 2}
$$


where the anisotropic part of the sub-filter tensor is denoted by $\tau_{i j}^{a}$ and the anisotropic part of the resolved stress tensor by $R_{i j}^{a}=\bar{u}_{i}^{\prime} \bar{u}_{j}^{\prime}-\bar{u}_{k}^{\prime} \bar{u}_{k}^{\prime} \delta_{i j} / 3$. In case of an eddy-viscosity model the anisotropic sub-filter tensor $\tau_{i j}^{a}=-2 \nu_{s f s} \bar{S}_{i j} \quad$ with $\quad \nu_{s f s}=\left(C_{k} L^{2}\right)|\bar{S}| . \quad$ This model implies

$$
\begin{array}{r}
\left\langle\tau_{i j}^{a} \tau_{i j}^{a}\right\rangle=4\left\langle\nu_{s f s} S_{i j} S_{i j}\right\rangle=\left\langle 2 L^{4}|S|^{4}\right\rangle C_{k}^{4} \equiv \chi_{1} C_{k}^{4} \\
\left\langle\tau_{i j}^{a} R_{i j}^{a}\right\rangle=\left\langle-2 \nu_{s f s} S_{i j} R_{i j}^{a}\right\rangle=-\left\langle 2 L^{2}|S| S_{i j} R_{i j}^{a}\right\rangle C_{k}^{2} \equiv \chi_{2} C_{k}^{2}
\end{array}
$$

If we denote in addition $\left\langle R_{i j}^{a} R_{i j}^{a}\right\rangle \equiv \chi_{3}$ then we infer a fourth order polynomial equation governing the effective model coefficient in terms of 'local' averages of LES resolved quantities as

$$
\chi_{1}\left(1-\frac{1}{s_{\tau}^{2}}\right) C_{k}^{4}+2 \chi_{2} C_{k}^{2}+\chi_{3}=0
$$

from which the unknown coefficient $C_{k}$ can be obtained once the desired sub-filter activity is set to an appropriate value.

In local ILSA the averaging operations in $L$ and $s_{\tau}$ is carried out over time. Results for a number of flows indicated that an averaging time window comparable to the integral time scale of the flow or larger yields results similar to the case in which averaging over homogeneous directions is adopted. This underlines the model's robustness in heterogeneous cases. Furthermore, the advantage of $s_{\tau}$ over $s_{\varepsilon}$ lies in its applicability to high Reynolds number flows where $s_{\varepsilon}$ asymptotes to unity and becomes insensitive to the choice of $C_{k}$ (Piomelli et al. 2015).

The key innovation of both ILSA models is in the fact that the user may specify the level of LES resolution in terms of the sub-filter activity $s_{\varepsilon}$ or $s_{\tau}$. To compare local ILSA to global ILSA, application of both models to turbulent flow in a plane channel will be considered in the next Section.

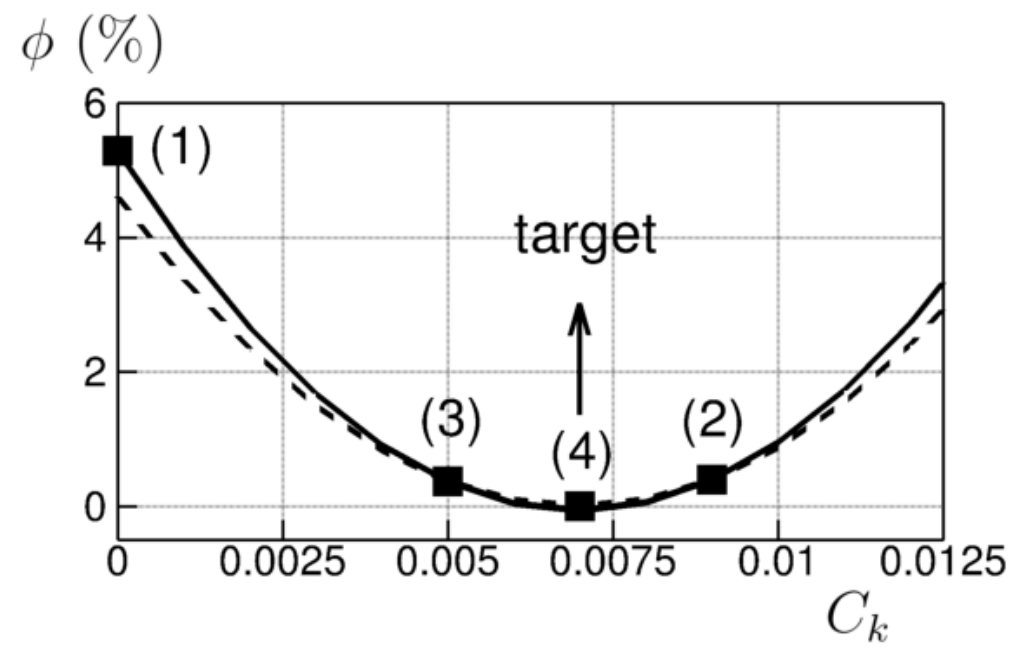

Figure 2: Convergence of the SIPI approach yielding an effective model coefficient to achieve a dissipation rate based sub-filter activity $s_{\varepsilon}=0.23$ : (solid) parabola through (1) (2) and (3); (dashed) parabola through (2), (3) and (4). This illustration is for turbulent channel flow at $R e_{\tau}=950$ and a coarse spatial resolution of $48 \times 65 \times 48$ points. 
The development and application of the global and local ILSA models has been approached through a study of the classical turbulent flow configuration of a plane channel at moderate Reynolds numbers. This poses essential challenges to the new modeling approach as both turbulent flow in the core of the channel as well as in the boundary layers needs to be properly captured. We closely follow Piomelli et al., (2015) and Rouhi et al., (2016) describing recent progress regarding the set-up and application of the ILSA model for this flow. We first consider the global ILSA approach and subsequently discuss the achievements using the local ILSA method.

We consider a turbulent channel flow and adopt as measure for the sub-filter activity the ratio of the sub-filter scale - and total dissipation rates $s_{\varepsilon}$. A first step toward a successful application of the global ILSA model is the determination of the effective model coefficient. Following the SIPI approach one may vary this coefficient in coarse grid simulations such that a desired sub-filter activity level is achieved. In Figure 2 we show the distance to the desired sub-filter activity as function of $C_{k}$

$$
\phi_{s}=100 \times\left|s_{\varepsilon}-s_{t g t}\right|^{2}
$$

where we adopted a target value $s_{\mathcal{E}}=0.23$, corresponding to a well-resolved LES. We observe a rapid convergence of the effective model parameter to a value around 0.007 , using local parabolic reconstructions of the error landscape. At this effective model parameter the convergence of turbulence statistics at a range of increasing spatial resolutions can subsequently be inferred. This is illustrated in Figure 3. There is a clear convergence to grid-independent LES predictions at spatial resolutions that are a fraction of the resolutions required for a genuine DNS. The mean velocity profile as well as velocity fluctuations all converge smoothly to the corresponding DNS results. We also applied this global ILSA method to turbulent channel flow at much higher Reynolds number, $R e_{\tau}=2000$. In this case, the SIPI optimization on a coarse grid yields an optimal effective model coefficient around 0.005. This shows a slight dependence of the optimal effective model coefficient on flow conditions, in line with the optimal refinement illustrated in Figure 1. 
Figure 3: Local ILSA model. Turbulence statistics for $R e_{\tau}=950$ and effective model coefficient

0.007 showing (a) mean velocity; (b) turbulent kinetic energy; (c) Reynolds shear stress, comparing to DNS data (+) of Hoyas and Jiménez (2006) at different resolutions:

$48 \times 65 \times 48($ long dash $), 64 \times 97 \times 64($ dash - dot $), 128 \times 129 \times 128($ dash $), 192 \times 193 \times 192($ solid $)$

In Piomelli et al., (2015) the role of the specific measure used for quantifying the sub-filter activity was scrutinized. Rather than adopting the dissipation rate based sub-filter activity, at sufficiently low Reynolds numbers, one may also adhere to other measures, e.g., based on the turbulent stresses or the shear stress. It was established that findings obtained with a particular measure could be translated into basically equivalent findings using one of the other measures, provided the correct corresponding target value for the sub-filter activity $s_{\varepsilon}$ is chosen. As an example, a target value of $s_{\varepsilon}=0.23$ for the dissipation-rate-based measure was found to be basically equivalent to a target value of around $s_{\tau}=0.022$ for the sub-filter stress based measure. Moreover, it was shown that the particular value for the effective model coefficient was not too sensitive for turbulent channel flow, showing that 'nearby' values can be used as well without unduly deteriorating the simulation results.

At appropriate values of the target sub-filter activity, the global ILSA model was shown to yield smoothly converging, grid-independent turbulent flow predictions that accurately correspond to 
available DNS data at a target sub-filter activity value of $s_{\varepsilon}=0.23$. To achieve such a result a number of steps need to be taken, among which the execution of several coarse grid ILSA simulations to obtain a good approximation of the optimal effective model coefficient. Obviously, this collection of coarse-grid precursor simulations is an undesired overhead that makes application of the global ILSA model more difficult. Therefore, we proceed by describing the 'local ILSA' model which adds to the robustness of the model, particularly at high Reynolds numbers, and removes the need for the prior coarse-grid calculations.
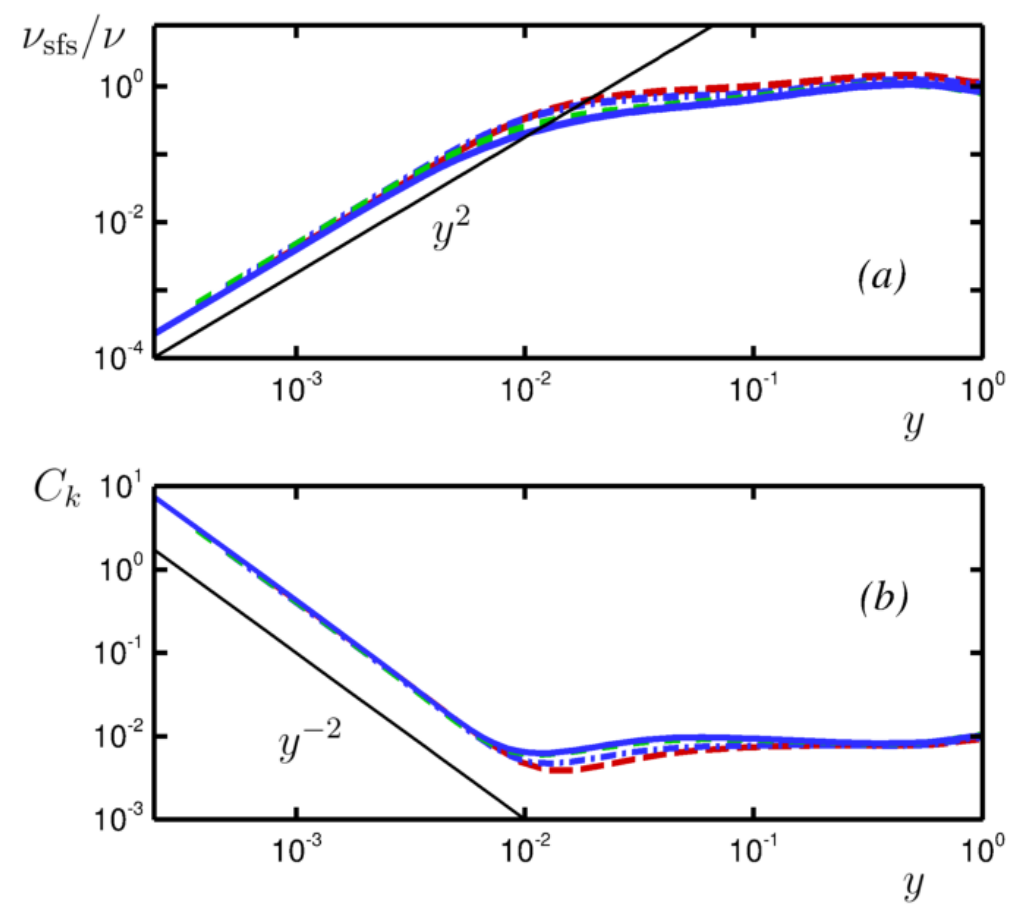

Figure 4: Profiles of $(a)$ the normalized sub-filter stress eddy-viscosity and $(b)$ the effective model coefficient for the local ILSA model at $R e_{\tau}=950$ with $s \tau=0.022$ and spatial resolutions $48 \times 65 \times 48($ long dash $), 64 \times 97 \times 64($ dash - dot $), 128 \times 129 \times 128($ dash $), 192 \times 193 \times 192($ solid $)$

We use the stress based sub-filter activity measure and adopt a target value of 0.022 for Reynolds number $R e_{\tau}=950$. The local ILSA model was found to yield similar or improved accuracy compared to the global ILSA model, at lower computational costs. Local ILSA findings also showed smooth convergence with increasing spatial resolution and good robustness with respect to small changes in the target value for the sub-filter activity. 
In Figure 4 we show in some more detail how the local ILSA model achieves its predictions. In Figure 4(a), close to the wall, the sub-filter eddy-viscosity shows quadratic scaling with the wallnormal distance. Even though this scaling deviates from the theoretical boundary layer result (cubic scaling), the fact that the eddy-viscosity approaches 0 near the wall is essential to avoid too much dissipation. This is a pre-requisite to avoid a qualitatively wrong prediction of the turbulent dynamics, as would be seen with the original Smagorinsky model in a boundary layer. Further away, in the bulk, the ratio of the turbulent to the molecular viscosity is quite constant and approaches unity, in case the spatial resolution is high enough to yield near grid independence. In Figure $4(b)$ the effective model coefficient shows a corresponding scaling inversely proportional to the square of the wall-normal distance. Further out in the bulk this model coefficient becomes quite constant as well.

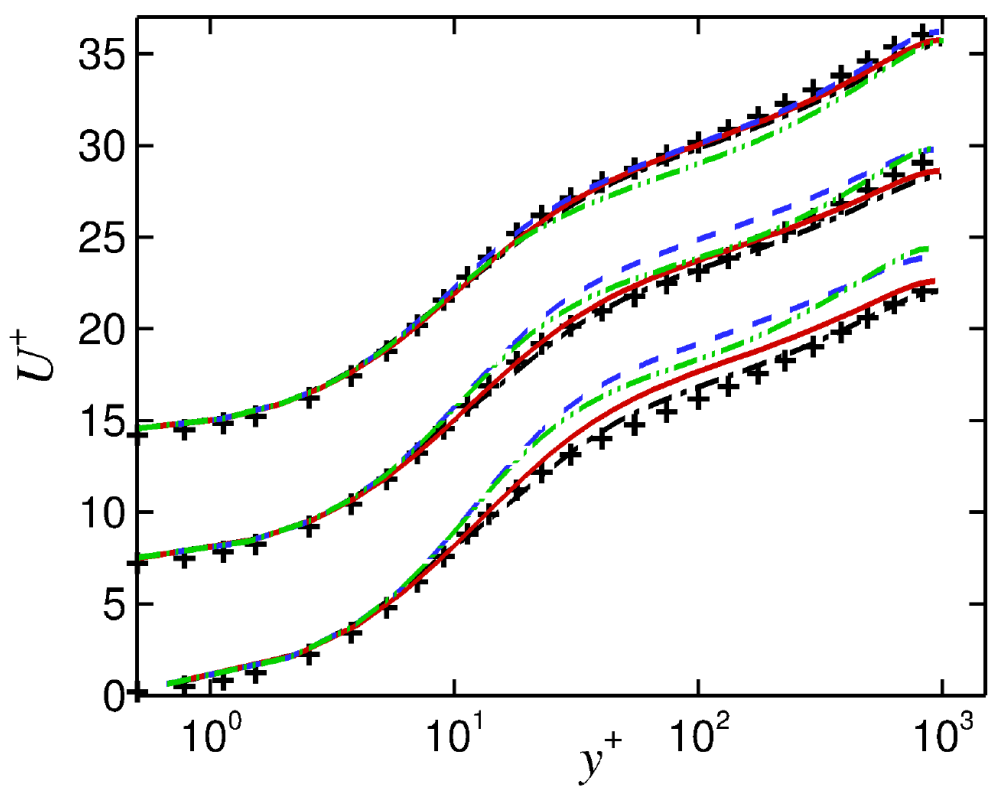

Figure 5: Mean velocity profiles at $R e_{\tau}=950$ comparing DNS data (+) with the dynamic model (dash), local ILSA (solid), global ILSA (dash-dot), both with with $s \tau=0.022$, and no model (dash-dot-dot) at three different spatial resolutions. Bottom curves: $48 \times 65 \times 48$ points; middle curves: $64 \times 97 \times 64$ points; top curves: $128 \times 129 \times 128$ points (reproduced with permission from Rouhi et al, 2016).

In Figure 5 we collect results comparing the two ILSA models with the dynamic model (Germano et al., 1991). Note that, in local ILSA $s \tau=0.022$ is assigned locally while in global ILSA $s \tau=$ 0.022 is assigned globally. We observe at coarse resolutions that all SFS models give improvements over a coarse DNS (no model used). The ILSA predictions show a close agreement with DNS data at a fraction of the computational cost of the dynamic model. With increasing spatial resolution, the variation among the predictions decrease, as expected, in view of the reduced influence of discretization errors. Global and local ILSA were found to require about the same computational resources, within $1 \%$ variation, as the 'no-model' option, while the implementation of the dynamic model required about $20 \%$ more computing time.

In summary, we have shown that both the global and local ILSA models yield accurate predictions of turbulent channel flow at high Reynolds numbers. The predictions are robust with respect to the precise value of the effective model coefficient, as long as it is taken within about $20 \%$ of the optimal value obtained using coarse grid simulations in the SIPI approach. The local ILSA model 
avoids pre-cursor simulations and is a significant improvement over the global ILSA model. We adopt the local ILSA model in a study of turbulent flow over a backward-facing step in the next Section.

\section{Local ILSA for flow over a backward-facing step}

In this Section we illustrate the performance of the local ILSA model for turbulent flow over a backward-facing step at $R e_{c}=U_{c} h_{S} / \nu=28,000$ based on the centerline velocity $U_{c}$ at the inlet $(x=0)$ and step height $h_{s}$.

We compare results with the Lagrangian dynamic model (Meneveau et al., 1996), and show close agreement of local ILSA with experimental reference data by (Vogel \& Eaton, 1985). We analyze the induced eddy-viscosity model on the computational grid and argue better numerical behavior in the ILSA model, contributing to the overall model performance. We also compare the reattachment length predictions for these simulations, a quantity of considerable relevance for assessing the performance of separated flow models. Finally, we present flow structures characterizing the turbulent mixing in the flow (Geurts, 2001) and show that the grid-independent LES predictions can be represented well provided minimal requirements are met by the grid.

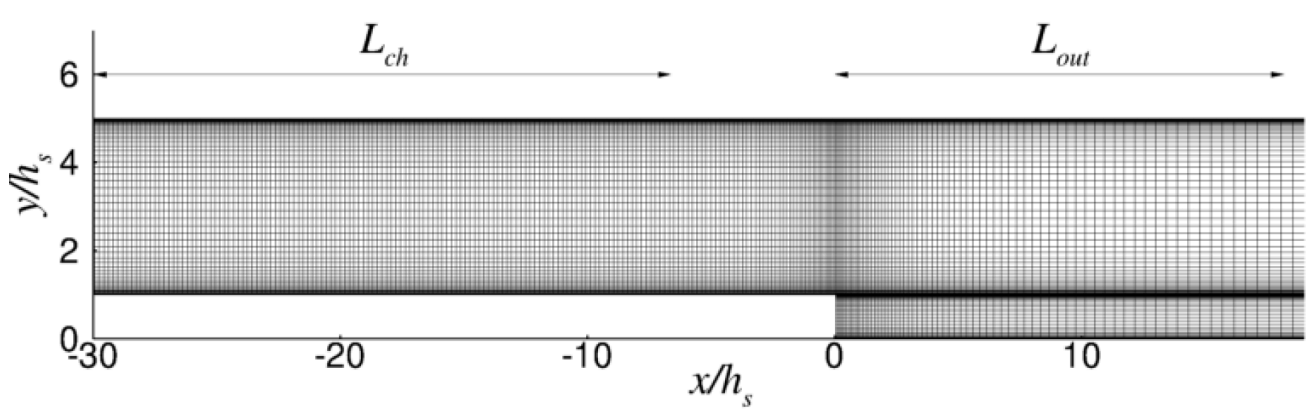

Figure 6: Structured grid for the backward-facing step flow on a coarse grid of $256 \times 100 \times 64$ grid points, clustered at characteristic locations in the domain, i.e., near the boundaries and intense

shear layers inside the domain. All scales are normalized by the step height $h_{s}$.

In Figure 6 we show the computational grid used for the backward-facing step simulations. The height of the inflow channel is 4 step heights and the spanwise width is 3 step heights. The inflow length of the channel is 32 step heights and the velocity field at $x=-5 h_{S}$ is recycled to the inflow located at $\mathrm{x}=-32 h_{s}$ to generate a well-developed turbulent inflow. This was validated separately by comparison with a turbulent channel flow. At the outflow at $20 h_{s}$ a convective boundary condition was adopted. Comparison with a longer domain with an outflow at $30 h_{s}$ confirmed that the domain is adequately long.

In Figure 7 the mean flow statistics are shown at three spatial resolutions, comparing local ILSA with the Lagrangian dynamic model, with 'no model' and with experimental data. The LES results agree closely with each other and with the experimental data - only on the coarsest grid there is a slight difference between the local ILSA and Lagrangian dynamic model. This difference is most notable in the recovery region after the reattachment. The 'no model' option shows that the inclusion of a proper eddy-viscosity model is beneficial for the accuracy of 
predictions, even though the spatial grid is highly refined. Similar results are obtained for the Reynolds stresses (not shown).
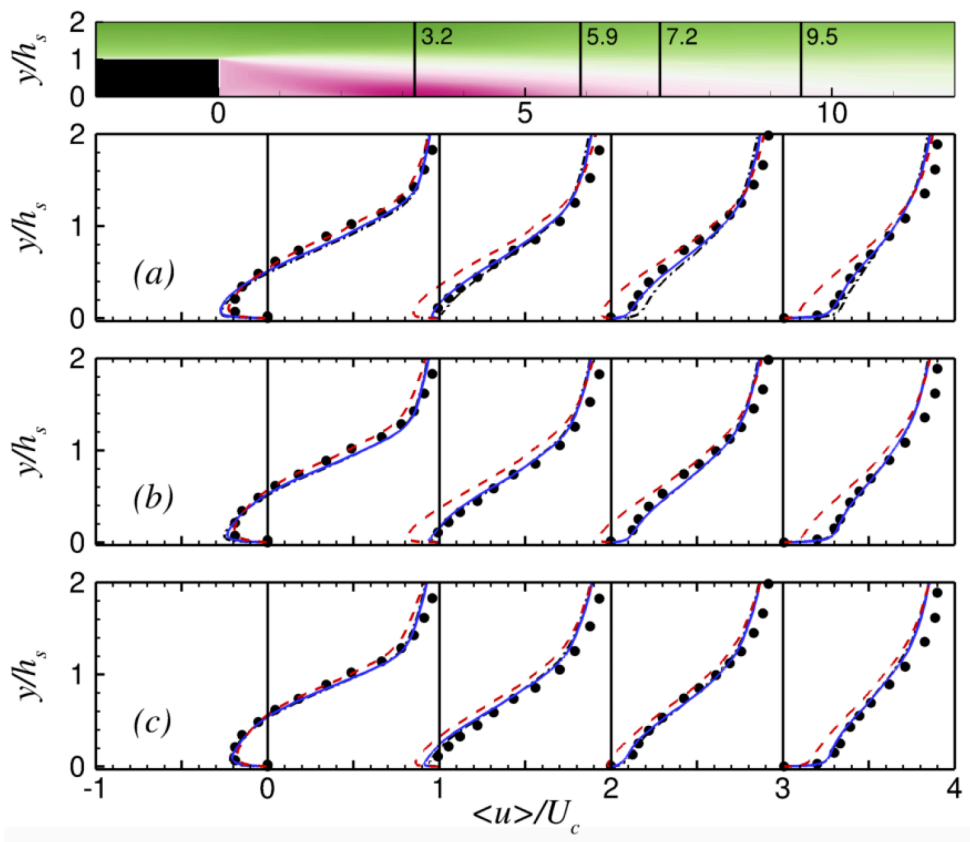

Figure 7: Mean velocity nrmalized by the centerline velocity at the inlet, determined at a number of locations downstream of the step on different grids: (a) $256 \times 100 \times 64$ points, (b) $384 \times 150 \times 96$ points, (c) $512 \times 200 \times 128$ points. Experimental data (Vogel \& Eaton, 1985) shown with full circles, Lagrangian dynamic model in dash-dot, no-model in dashed line and local ILSA in solid line (reproduced with permission from Rouhi et al., 2016).

The central model parameters of the local ILSA model are illustrated in Figure 8. We compare the standard definition of the filter width, $\Delta=(\Delta x \Delta y \Delta z)^{1 / 3}$ (Figure $8 a$ ) with the estimated integral scale $L$ (Figure $8 b$ ). The local integral length-scale decreases considerably where the flow has small scale features, i.e., in the boundary layers and near the shear layers. Away from these locations, $L$ increases as the typical scales that need resolving become larger. Although the grid tries to reproduce some of these features, its structured character implies that a refined mesh is also used in regions where the turbulent eddies are not small, for instance downstream of the step, $x / h_{s} \simeq 5-10$ and $y / h_{s} \simeq 1$. As a consequence, the eddy viscosity predicted by the Lagrangian eddy viscosity model (which depends on $\Delta^{2}$ ) has an unphysical sharpness along the region where the grid is refined (Figure $8 d$ ), which is not observed when the local ILSA model is used (Figure $8 c$ ). Such large variations in the local filter-width and eddy viscosity are linked to commutator errors (Van der Bos \& Geurts, 2005; Vanella et al., 2008) that thus far have not been accounted for. By allowing a smooth variation of the eddy-viscosity/filter-width, the contribution of commutator errors can largely be removed (Van der Bos \& Geurts, 2005, Geurts \& Holm, 2006). 

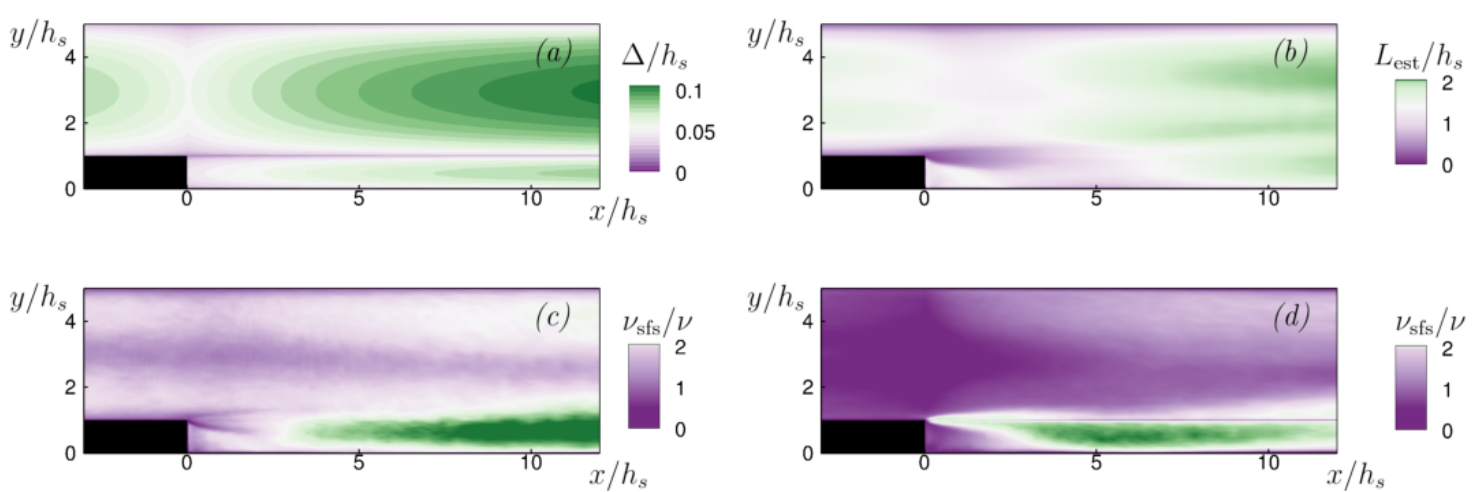

Figure 8: SFS quantities for the backward-facing step flow. (a) Filter size; (b) integral scale; $(c)$ eddy viscosity, Local ILSA model; $(d)$ eddy viscosity, dynamic Lagrangian model. Intermediate grid, $384 \times 150 \times 96$ points.

As a further assessment of the quality of the LES predictions we consider the distribution of the skin friction defined as

$$
C_{f}=\frac{2 \tau_{w}}{\rho U_{c}^{2}}
$$

in terms of the wall-shear stress, the fluid density and the centerline velocity, and the reattachment length $X_{r}$, given by the length behind the step at which the time-averaged wall-shear stress changes sign. The simulations reported above are modeled after experiments by Vogel and Eaton (1985). Skin friction and re-attachment length can be directly compared to the experimental values. In Figure 9(a) we observe that the general trend of the skin friction is well captured by the Lagrangian dynamic model and the local ILSA model when using the previously determined optimal value of 0.022 for the sub-filter activity. The error in the skin friction is quite significant in case the 'no model' option is considered, i.e., at zero sub-filter activity. The error also increases when too large values of the sub-filter activity are adopted, establishing that 0.022 is a sensible value for this flow. In Figure 9 (b) both the Lagrangian dynamic model and the local ILSA model are seen to agree closely with each other and with the measured skin friction distribution.

In Figure 11, the re-attachment length predictions obtained with the local ILSA model are compared with the Lagrangian dynamic model predictions and the experimental value. The experimental value is accurate to an estimated relative error of about 10\% (Vogel \& Eaton, 1985). The Lagrangian dynamic value is seen to lie within the experimental range. This is also observed for the local ILSA model with sub-filter activity levels between 0.015 and 0.04 . For the optimal sub-filter activity level of 0.022 , agreement is indeed very close to the reported experimental value. The 'no-model' option is seen to deviate beyond the experimental uncertainty from the experimental value. Increasing the spatial resolution was not found to yield significant changes. 

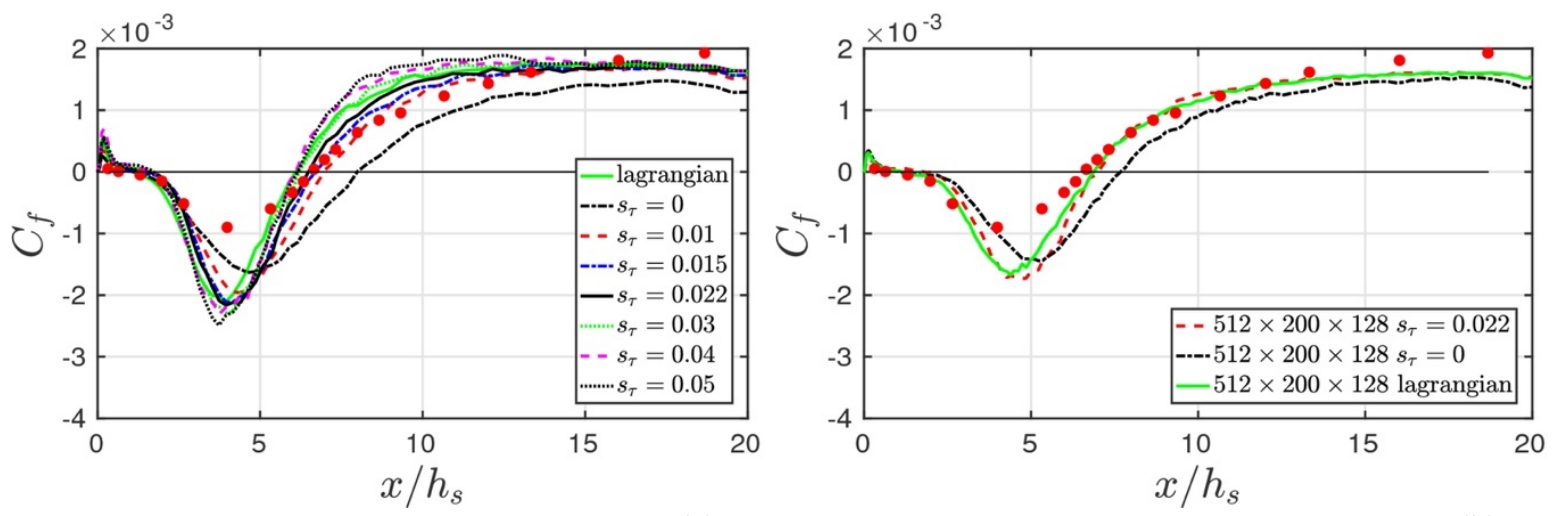

(a)

(b)

Figure 9: Skin friction distribution obtained with the local ILSA model at a range of specified sub-filter activity levels (a) and a comparison between local ILSA and Lagrangian dynamic model predictions (b).

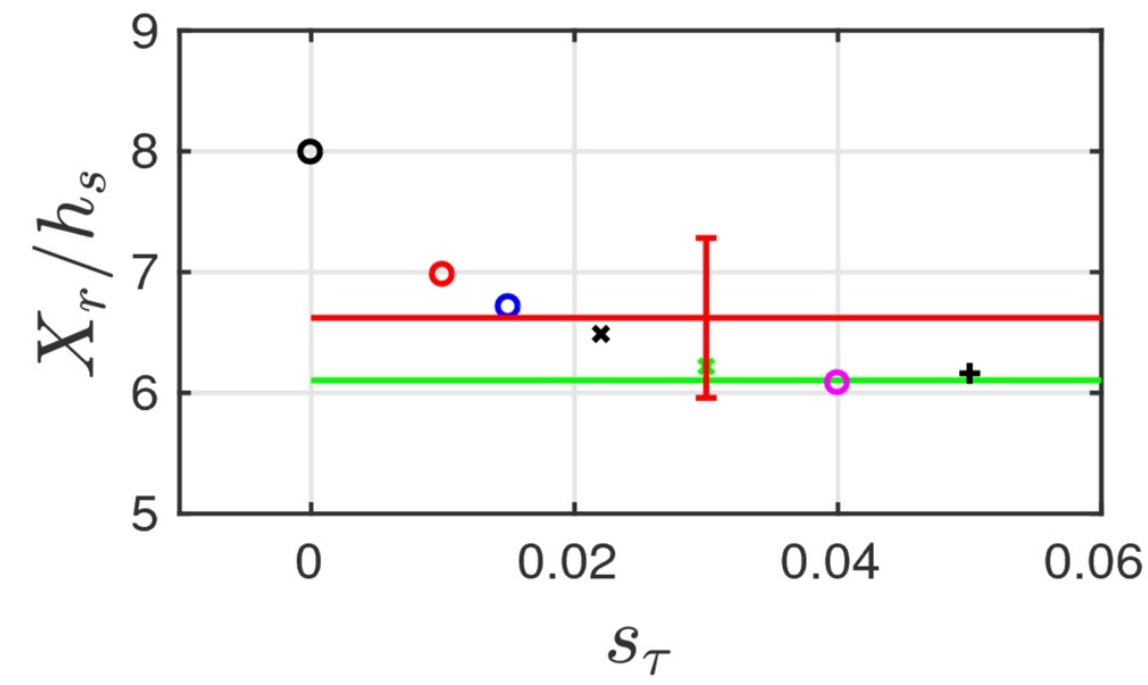

Figure 10: Re-attachment length as function of the imposed sub-filter activity level for the local ILSA model. Predictions are compared with the Lagrangian dynamic model (green horizontal line) and the experimental value (red horizontal line with $10 \%$ experimental uncertainty).

The effects of spatial resolution on the structures in the numerical solution can be inferred qualitatively from three-dimensional snapshots of characteristic flow properties. Figure 11 shows contours of the streamwise velocity fluctuations obtained on three different grids. One may observe that the coarse mesh is not sufficiently refined to resolve eddies of the size of the effective filter-width, while the two finer grids yield a qualitatively similar impression of the flow structures. Indeed, there is very little difference between the results obtained at the two finer meshes - the predicted flow does not change much with resolution, as the integral scale is wellapproximated throughout the domain on both finer grids. 


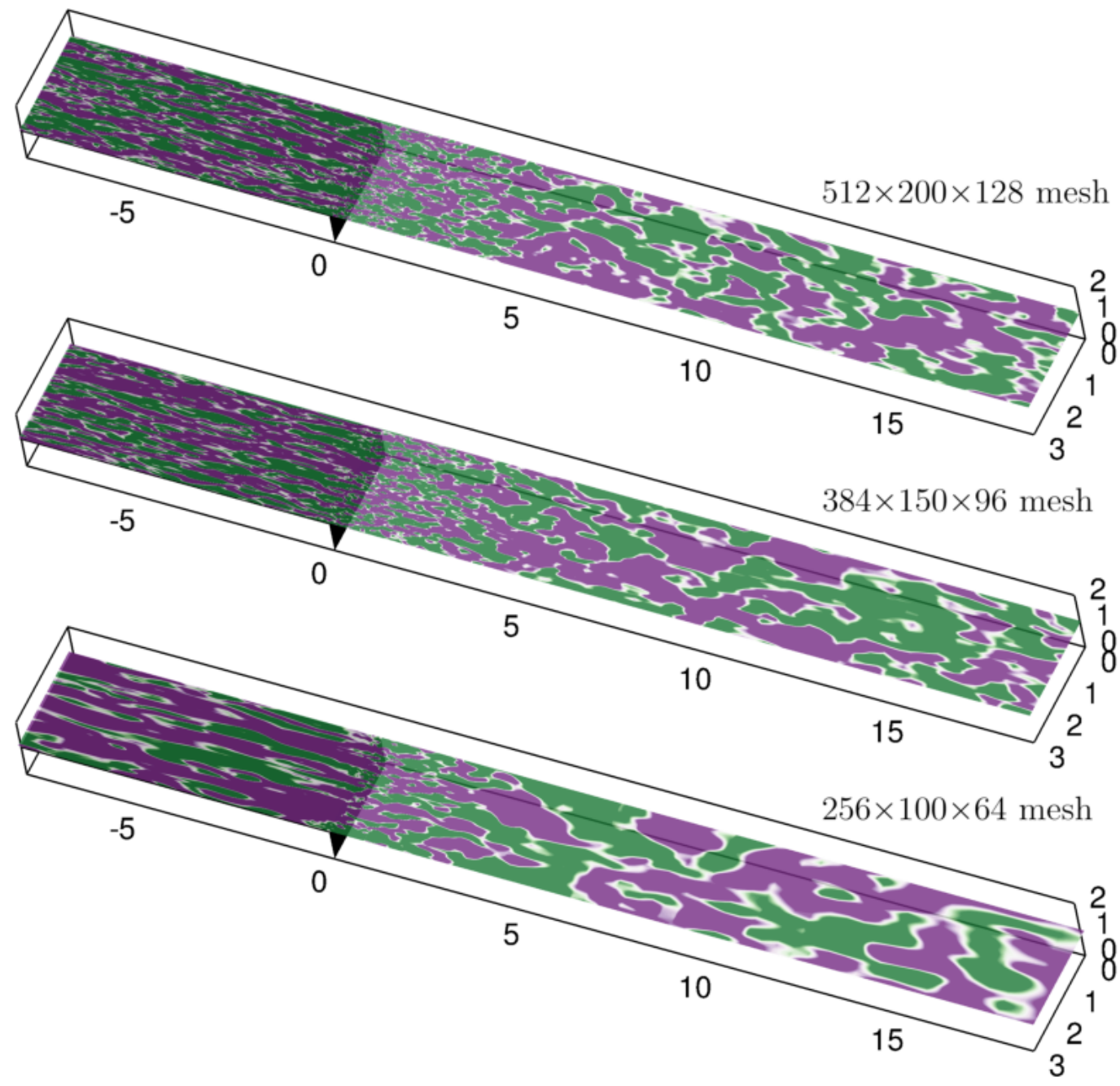

Figure 11: Contours of $u$-velocity fluctuations, normalized by the centerline velocity $U_{c}$ at fixed vertical height, using three different meshes of increasing refinement.

In figure 12 we visualize the eddies using isosurfaces of the second invariant of the velocity gradient tensor, Q (Hunt et al., 1988; Dubief \& Delcayre, 2000):

$$
Q=-\frac{1}{2} \frac{\partial \bar{u}_{i}}{\partial x_{j}} \frac{\partial \bar{u}_{j}}{\partial x_{i}}
$$

We observe a marked qualitative difference between the impression of $\mathrm{Q}$ on the coarse mesh, compared to that seen on the two finer grids. With increased resolution, from the middle grid onward, the visual impression of the flow starts to appear quite similar. This intuitively indicates a level of convergence to a grid-independent LES. 


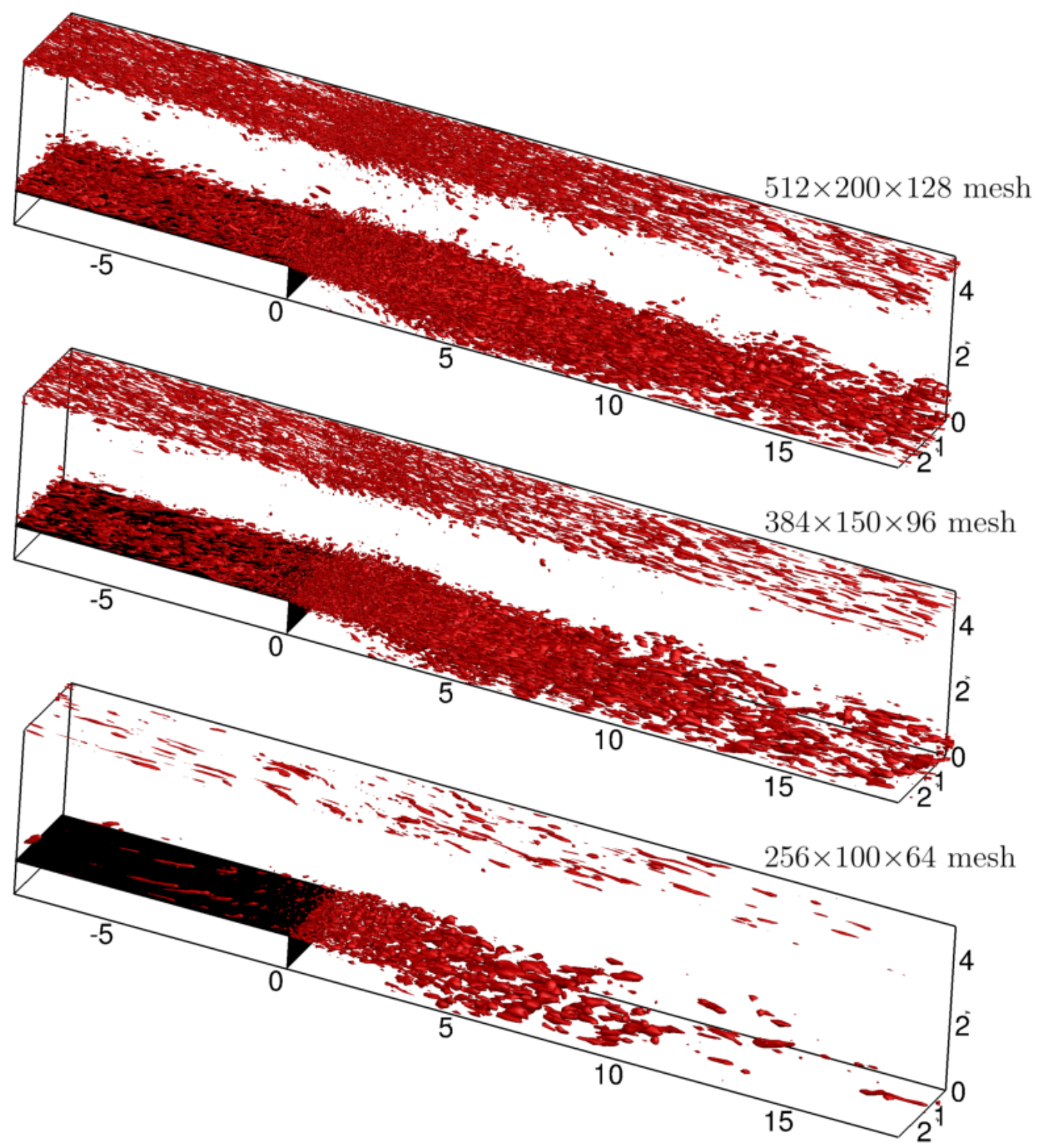

Figure 12: Isosurfaces of the second invariant of the velocity gradient tensor $Q$ using three different meshes. Top: fine mesh; middle: medium mesh; bottom: coarse mesh.

In summary, application of the local ILSA model to flow over a backward-facing step shows better control over the smoothness of the spatial distribution of the eddy-viscosity, compared to the Lagrangian dynamic model. This is beneficial for the numerical accuracy - overall a close agreement with experimental data is achieved by inclusion of flow-based length-scale variations, at a much reduced computational cost.

\section{Concluding remarks}

We reviewed recent progress in the assessment of the reliability of LES predictions. The basic limitation in LES quality stems from an interplay between effects of discretization errors and modeling error. This can be clarified comprehensively in terms of a computed error-landscape in which the total simulation error is computed as function of spatial resolution and model coefficient. Such an approach yields an insight in the total error after a large number of 
simulations has been conducted and compared to a 'ground truth', e.g., DNS data or experimental findings.

A key concept used for dynamic error control for LES in this paper is the 'sub-filter activity'. This measures the dynamic relevance of scales that were removed from the dynamics through spatial filtering. Depending on whether 'a lot' of small scales were removed during coarsening or not, the main source of total simulation error may vary from that of being dominated by sub-filter modeling error to that of being dominated by spatial discretization error. Adhering to a description that keeps the measure for the sub-filter activity near a pre-specified target value, allows some level of control over these dominant LES errors.

In this paper we combined both 'error-landscape' and 'sub-filter activity' concepts into the global and local ILSA models. The coarsening length-scale was based on the integral length scale of the flow, rather than on the local grid scale. We used the SIPI method to optimize the effective model coefficient such that a target sub-filter activity is achieved. This requires a number of coarse grid simulations, yielding a good approximation of the desired effective model coefficient. The multiple resolution approach, i.e., first performing coarse simulations to estimate the required effective model coefficient, followed by higher resolution simulations to predict the flow accurately, is basic to the original 'global ILSA' model (Piomelli et al., 2015). By using a subfilter measure based on the unresolved stresses, a 'local ILSA' model can be formulated as well, in which the pre-cursor simulations can be avoided altogether.

We tested the local and global ILSA models extensively in plane channel flow at high Reynolds numbers and in a backward-facing step flow. The ILSA models were found to require little extra computational overhead and to yield close agreement with DNS and experimental reference material. The models are quite robust and the prediction quality is not particularly sensitive to the precise value of the effective model coefficient. The main model innovation, i.e., that of using the local integral length scale to represent changes in the local flow physics, rather than using the local grid spacing, represents the 'dynamic' aspect in the ILSA models. Much of the non-uniform variations in the turbulence properties is already reflected in changes in the integral length scale - the rest of the eddy-viscosity definition is then less sensitive to flow details and was found to yield accurate simulations and a natural adaptation of the sub-filter model to main features of the flow. This was observed in boundary layers, and in regions of high shear such as in the backwardfacing step configuration.

The local ILSA model holds promise to be effective in LES also for wider classes of turbulent flow. Further studies to underpin this should include stronger variations in flow properties, including re-laminarization. Moreover, investigating the role of the target value for the sub-filter activity level on the reliability of the LES predictions and the convergence with spatial resolution are items of ongoing research toward a genuine error-bar for CFD.

\section{References}

Smagorinsky, J., 1963, General circulation experiments with the primitive equations. I. The basic experiment. Mon. Weath. Rev. 91,99-164

Schumann, U., 1975, Subgrid scale model for finite difference simulations of turbulent flows in plane channels and annuli, J. Comput. Phys, 18, 376-404

Pope, S. B., 2000 Turbulent Flows. Cambridge University Press, ISBN 978-0521598866

Geurts, B. J. 2003, Elements of Direct and Large-Eddy Simulation. R.T. Edwards Inc, ISBN 978-1930217072

Piomelli, U., Rouhi, A., Geurts, B.J., 2015, A grid-independent length scale for large-eddy simulations 
Journal of fluid mechanics $766,499-527$

Rouhi, A., Piomelli, U., Geurts, B.J., 2016, Dynamic subfilter-scale stress model for large-eddy simulations Physical Review Fluids 1 (4), 044401

Meyers, J., Geurts, B.J., Baelmans, M., 2003, Database analysis of errors in large-eddy simulation, Physics of Fluids $15(9), 2740-2755$

Meyers, J., Geurts, B.J., Sagaut, P., 2007, A computational error-assessment of central finite-volume discretizations in large-eddy simulation using a Smagorinsky model, Journal of Computational Physics 227 (1), 156-173

Meyers, J., Meneveau, C., Geurts, B.J., 2010, Error-landscape-based multiobjective calibration of the Smagorinsky eddy-viscosity using high-Reynolds-number decaying turbulence data, Physics of fluids 22 (12), 125106

Vreman, B., Geurts, B.J., Kuerten, H., 1994, Discretization error dominance over subgrid terms in large eddy simulation of compressible shear layers in 2D, Communications in numerical methods in engineering 10 (10), 785-790

Meyers, J., Sagaut, P., Geurts, B.J., 2006, Optimal model parameters for multi-objective large-eddy simulations, Physics of Fluids 18 (9), 095103

Geurts, B.J., 2006, Interacting errors in large-eddy simulation: a review of recent developments, Journal of turbulence, N55

Boersma, B.J., Kooper, M.N., Nieuwstadt, T.T.M., Wesseling, P., 1997, Local grid refinement in large-eddy simulation, Journal of Engineering Mathematics 32 (2-3), 161-175

Piomelli, U., Geurts, B.J., 2010, A grid-independent length scale for large-eddy simulations of wall-bounded flows. In Proceedings of 8th International Symposium Engineering Turbulence Modelling and Measurements - ETMM8 (eds. M. A. Leschziner, P. Bontoux, B. J. Geurts, B. E. Launder \& C. Tropea), pp. 226-231

Geurts, B.J., Fröhlich, J., 2002, A framework for predicting accuracy limitations in large-eddy simulation. Phys. Fluids 14 (6), L41-L44.

Geurts, B.J., Meyers, J., 2006, Successive inverse polynomial interpolation to optimize Smagorinsky's model for largeeddy simulation of homogeneous turbulence, Physics of fluids 18 (11), 118102

Vogel, J.C., Eaton, J.K., 1985, Combined heat transfer and fluid dynamic measurements downstream of a backwardfacing step, ASME: Journal of Heat Transfer. 107, 4, 922-929

Geurts, B.J., 1999, Balancing errors in LES, Direct and Large-Eddy Simulation III, 1-12

F. Nicoud, J. S. Baggett, P. Moin, W. H. Cabot. Large eddy simulation wall-modeling based on subop- timal control theory and linear stochastic estimation. Phys. Fluids, 13:2968-2984, 2001.

Geurts, B.J., 2002, How can we make LES fulfill its promise? Advances in LES of complex flows, 13-32

Geurts, B.J., Holm, D.D., 2003, Regularization modeling for large-eddy simulation, Physics of fluids 15 (1), L13-L16

Bardina, J., Ferziger,, J. H. \& Rogallo, R. S. 1980 Improved subgrid scale models for large- eddy simulation. AIAA Paper 80-1357.

Van der Bos, F., Van der Vegt, J.J.W., Geurts, B.J., 2007, A multi-scale formulation for compressible turbulent flows suitable for general variational discretization techniques, Computer methods in applied mechanics and engineering 196 (29-30), 2863-2875

Geurts, B.J., Van der Bos, F., 2005, Numerically induced high-pass dynamics in large-eddy simulation, Physics of fluids 17 (12), 125103

Van der Bos, Geurts, B.J., 2010, Computational error-analysis of a discontinuous Galerkin discretization applied to large-eddy simulation of homogeneous turbulence, Computer methods in applied mechanics and engineering 199 (1316), 903-915

Klein, M., Meyers, J., Geurts, B.J., 2008, Assessment of LES quality measures using the error landscape approach, Quality and Reliability of Large-Eddy Simulations, 131-142 
Hoyas, S., Jiménez, J., 2006, Scaling of the velocity fluctuations in turbulent channels up to $\mathrm{Re}_{-}=2003$, Physics of Fluids. 18, 1, 011702

Germano, M., Piomelli, U., Moin, P., Cabot, W.H., 1991, A dynamic subgrid-scale eddy viscosity model, Physics of Fluids A: Fluid Dynamics 3 (7), 1760-1765

Meneveau, C., Lund, T.S., Cabot. W.H., 1996, A Lagrangian dynamic subgrid-scale model of turbulence, Journal of Fluid Mechanics, 319, 353-385

Geurts, B.J., 2001, Mixing efficiency in turbulent shear layers, J. Turbulence 2 (17), 1-23

Van der Bos, F., Geurts, B.J., 2005, Commutator errors in the filtering approach to large-eddy simulation, Physics of Fluids 17 (3), 035108

Vanella, M., Piomelli, U., Balaras, E., 2008, Effect of grid discontinuities on large-eddy simulation statistics and flow fields, Journal of Turbulence. 9

Van der Bos, F., Geurts, B.J., 2005, Lagrangian dynamics of commutator errors in large-eddy simulation, Physics of fluids 17 (7), 075101

Geurts, B.J., Holm, D.D., 2006, Commutator errors in large-eddy simulation, Journal of physics A: mathematical and general 39 (9), 2213

Hunt, J.C.R., Wray, A.A., Moin, P., 1988, Eddies, streams, and convergence zones in turbulent flows. In Studying Turbulence Using Numerical Simulation Databases, 2. Proceedings of the 1988 Summer Program, pp. 193-208. Stanford University.

Dubief, Y., Delcayre, F., 2000, On coherent vortex identification in turbulence. J. Turbul. 1, 011-1-22. 